TH E U N I VER I T Y O F M I C H I G A N COLLEGE OF LITERATURE, SCIENCE, AND THE ARTS

Department of Mathematics

Technical Report No. Il

FUNCTIONAL ANALYSIS AND BOUNDARY VALUE PROBLEMS

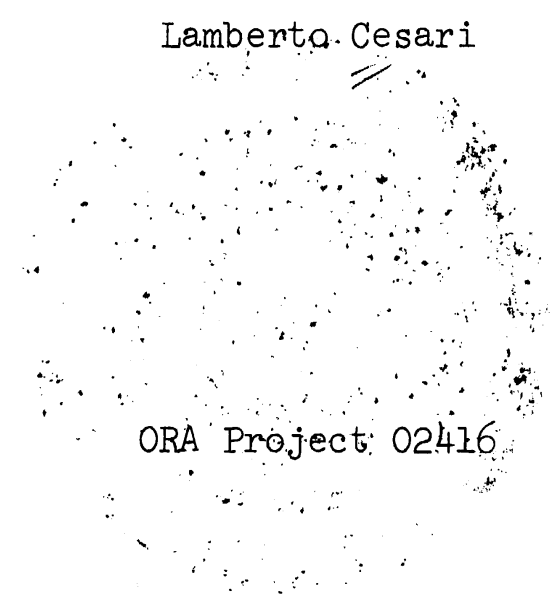

Submitted for:

UNITED STATES AIR FORCE

AIR FORCE OFFICE OF SCIENTIFIC RESEARCH

GRANT NO. AFOSR-69-1662

ARLINGTON, VIRGINIA

administered through:

OFFICE OF RESEARCH ADMINISTRATION ANN ARBOR

June 1970 
ENGP

UMRET2Z 


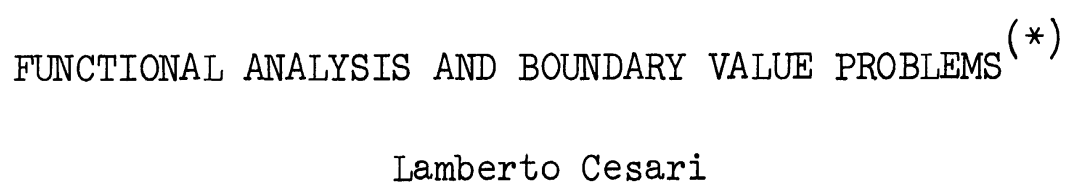

1. A Review of Research

1.1 EXISTENCE AND STABILITY OF PERIODIC SOLUTIONS OF PERTURBATION PROBLEMS In the years 1952-60 a great deal of work was done by a group of us (Cesari $[5 a, b, 1,6 a, b]$, R. A. Gambill $[2,9 a, b, c], J . K$. Hale $[10,12 a, b, c, d, e, f, g, h, i, j]$, and others) in relation to periodic solutions of perturbation problems for ordinary differential equations. This work was based on functional analysis, and particularly on fixed point theorems, projector operators, and bifurcation equations in terms of functional analysis.

We obtained criteria for the existence of periodic solutions (harmonic, subharmonics, ultraharmonic) of systems of periodic ordinary differential equations of the perturbation type

$$
\begin{gathered}
d u / d t=A u+\varepsilon f(t, u, \varepsilon), \\
u=\left(u_{1}, \ldots, u_{n}\right), f(t+T, u, \varepsilon)=f(t, u, \varepsilon),
\end{gathered}
$$

criteria for the existence of cycles of systems of autonomous equations

$$
d u / d t=A u+\varepsilon f(u, \varepsilon), u=\left(u_{1}, \ldots, u_{n}\right),
$$

*Research partially supported by US-AFOSR research grant 69-1662 at The University of Michigan. This paper has been read at the Symposium in Differential Equations at Western Michigan University, Kalamazoo, Michigan, April 30-May 2, 1970. 
and methods for the analysis of the asymptotic stability of periodic solutions, and of asymptotic orbital stability of cycles. For the particular purpose of a new and convergent method of successive approximations was devised.

In particular, J. K. Hale proved that nonlinear autonomous perturbation type systems of ordinary differential equations may easily present families of periodic solutions, or cycles, under suitable conditions of symmetry. J. K. Hale gave also simple criteria for this occurrence, and for the determination of the dimension of the family of cycles [12c, 5b].

I presented aspects of this first phase of the research in [5c] and in my book ([5d], pp. 66-78, and pp. 123-135). Halanay reported the method of successive approximations in his book ([11], pp. 308-317) together with the proof of its convergence and applications. J. K. Hale gave a detailed account of aspects of this first phase of the research in his books ([12k], pp. 27-94).

This work on periodic solutions of perturbation type problems in ordinary differential equations was then continued by C. Imaz [17], and by J. Mawhin $[2 l a, b, c, d]$. C. Imaz discussed particularly the case in which the underlying linear problem has multiple characteristic roots. J. Mawhin, in an extensive and systematic investigation still underway, has improved some of the results of J. K. Hale and R. A. Gambill.

In view of numerical analysis C. Banfi [3a,b] and C. Banfi and G. Casadei [4] considered a variant of the method of successive approximations, suited for high speed computers, and in which the actual successive approximations and the analysis and solution of the bifurcation equation are combined. They successfully experimented with the method. 


\subsection{BOUNDARY VALUE PROBLEMS FOR GENERAL NONLINEAR PROBLEMS}

In my paper [5e] I presented the underlying ideas of the method, in an abstract form, for general boundary value problems for ordinary and partial differential equations, even, strongly nonlinear. Also, I showed connections of the method with Galerkin's approach. In [5e] I essentially considered problems whose underlying linear formulation was selfadjoint. J. Locker [20a,b], and J. K. Hale, S. Bancroft, and D. Sweet [13] gave suitable extensions of the method to "nonselfadjoint" problems.

In [5f] I considered the problem of periodic solutions of systems of periodic ordinary differential equations nonnecessarily of the perturbation type, from the view point of the method as presented in [5c]. Also I proved in [5f] that the method contains as a particular case the much simpler approach used earlier for perturbation problems only. My work in [5f] was extended to problems of periodic solutions of ordinary differential equations with time lags by A. M. Rodionov [25].

S. A. Williams [27] showed that the method under consideration, as described in [5c], has a strict theoretical link with the Leray-Schauder approach [29]. Hale, Bancroft, and D. Sweet in their paper [13] emphasized other connections with previous or current work of D. C. Lewis, H. A. Antosiewicz, Jane Cronin, R. G. Bartle, L. Nirewberg, and Y. Sibuya.

I presented aspects of this second phase of the research in [5l]. Jane Cronin reported the main idea of the method, as presented in [5f] for periodic solutions only, in her book ([8], pp. 180-185). J. K. Hale gave a detailed account of aspects of this second phase of research in his books ([12k], pp. 9699, and [12m], pp. 252-290). 
For the particular problem of periodic solutions of ordinary differential equations, nonnecessarily of the perturbation type further theoretical work was done by H. W. Knobloch [18a] in connection with the use of the uniform norm, suitable estimates of the Fourier approximations, and a discussion of the bifurcation equations for any order of approximation by topological considerations based on C. Miranda's version of Bro wer's fixed joints theorem, version which had been already used in [5b]. H. W. Knobloch proved then simple existence theorems [18b] for periodic solutions of nonlinear periodic equations $y^{\prime \prime}+g\left(t, y, y^{\prime}\right)=0$, based only on qualitative properties of $g$, and this analysis led him to comparison and oscillation theorems for the same equations [18c]. In $[5 g, i, j]$ I studied periodic solutions in $x$ and $y$ of the partial differential equation

$$
u_{x y}=g\left(x, y, u, u_{x}, u_{y}\right)
$$

where $u$ is an $n$-vector, in the frame of the same method as presented in [5e]. By assuming $g$ continuous in all its argument and Lipschitzian in $u_{x}, u_{y}$, or in $u, u_{x}, u_{y}$, I obtained criteria for the existence of periodic solutions $u(x, y)$ continuous in $E_{2}$ with $u_{x}, u_{y}$ and $u_{x y}$. In [5h] I applied the same approach to the problem of solutions $u(x, y)$ to the same partial differential equation above, which are periodic with respect to $x$ only and continuous with $u_{x}, u_{y}, u_{x y}$ in a thin $\operatorname{strip}[-\infty<x<+\infty,|y| \leq a]$. The results of $[5 g, i, j]$ yield analogous results for the nonlinear wave equation

$$
u_{t t}-u_{x x}=g\left(t, x, u, u_{t}, u_{x}\right)
$$

By the use of the same method, based on [5f], and actually the same projection 
operators, more stringent quantitative estimates have been successively obtained by A. K. Aziz [Proc. Amer. Math. Soc. 17, 1966]. J. K. Hale [12l], by the same method applied directly to the nonlinear wave equation, and the use of a different choice of projector operators, obtained perspicuous criteria for doubly. periodic solutions in $E_{2}$. Recently D. Petrovanu [24a] has used the same method in the study of periodic solutions of the equation

$$
u_{x y z}=g\left(x, y, z, u, u_{x}, u_{y}, u_{z}\right)
$$

and in the study of the periodic solutions [24b] of the Tricomi system of equations

$$
u_{x}=g(x, y, u, v), \quad v_{y}=h(x, y, u, v)
$$

where $u$ and $v$ are $m$ - and $n$-vectors respectively.

A. Naparstek [22] has also used the same method for periodic distributional solutions of the nonlinear wave equation

$$
u_{t t}-\sigma^{2} u_{x x}=\varepsilon g(t, x, u)
$$

where $g$ is periodic (of period $2 \pi$ ) in $x$ and $y$, and either $\sigma$ is a rational number, or an irrational one of a known class of real numbers badly approximated by rationals and everywhere dense on the positive real line. The result extend to the problem of periodic solutions of equations of the form

$$
u_{t t}-\sigma^{2} u_{x x}=\varepsilon g\left(t, x, u, u_{t}, u_{x}\right)
$$

and required a great deal of mathematical investigation. The bifurcation equation is studied in this work in terms of the theory of monotone operators. 
In the same line W. S. Hall [14] has recently proved the existence of doubly periodic solutions of partial differential equations of the form

$$
u_{t t}+(-I)^{p} D_{x}^{2 p} u=\varepsilon f\left(t, x, u, u_{t}, u_{x}, \ldots, D_{x}^{p} u\right)
$$

in suitable Banach spaces of periodic functions and distributions. The deep analysis which was needed for this work includes a discussion of the bifurcation in the lines of Minty and Browder's theory on monotone operators, a discussion of suitable boundary conditions for the problem under consideration, and smoothness properties of the solutions.

E. M. Landesman and A. C. Lazer [19] have recently proved a general existence theorem for the Dirichlet problem for nonlinear partial differential equations of the form

$$
L u+a u+g(u)=h(x),
$$

where $L$ is a general second order selfadjoint uniformly elliptic operator in a domain G, with bounded measurable coefficients, where a is a positive constant, $h$ is a given function in $L_{2}(G)$, and $z$ is a monotone continuous real valued bounded functions on $E_{1}$. The very subtle proof makes use of a technique which has points of contact with the one of the method under consideration.

Cesari [5k] by the use of the process described in [5f], has discussed the existence of solutions $u(x, y)$ for the boundary value problem

$$
\Delta u=g(x, y, u) \text { for }(x, y) \in A, u=0 \text { for }(x, y) \in \partial A \text {, }
$$

where $A$ is the unit circle in the xy-plane, $\partial A$ its boundary, $g$ is measurable in 
in $\mathrm{x}, \mathrm{y}$ for every $\mathrm{u}$, and Lipschitzian in $\mathrm{u}$ for every $\mathrm{x}, \mathrm{y}$, and where the solutions $u(x, y)$ are required to be continuous in the closed circle $A U d A$, with continuous $u_{x}, u_{y}$ in the open circle $A$, and $\Delta u=\partial^{2} u / \partial x^{2}+\partial^{2} u / \partial y^{2}$ in the sense of the theory of distributions-is a measurable function in $\mathrm{A}$.

Recently, W. A. Harris, Y. Sibuya, and L. Weinberg [15] have used the same approach under consideration here to obtain new and extremely simple proofs of the classical theorems of Cauchy, Frobenius, Perron, and Lettenmeyer on linear ordinary differential equations and systems in the complex field. These authors are actually concerned with systems of the form

$$
z^{s_{i}} d u_{i} / d z=\sum_{j=1}^{n} a_{i j}(z) u_{j}, \quad i=1, \ldots, n,
$$

where $z=x+i y$, and the coefficients $a_{i j}$ are holomorphic functions of $z$ in $a$ complex neighborhood $\mathrm{V}=[|\mathrm{z}|<\delta]$ of the origin $\mathrm{z}=0$. In the regular case $\left(a l l s_{i}=0\right.$ ) the bifurcation equation is trivial, and the system has a fundamental system of holomorphic solutions in $\mathrm{V}$. In the regular singular case (all $\left.s_{i}=1\right)$ the bifurcation equation reduces essentially to the indicial equation, and the system has the expected number of solutions of the forms $z^{P} P(z), \rho$ complex, $\mathrm{P}(\mathrm{z})$ a convergent power series of $\mathrm{z}$. For $\mathrm{d}=\max \mathrm{s}_{i}<\mathrm{n}$, the given system has at least $\mathrm{n}$ - d solutions holomorphic in $\mathrm{V}$. 


\section{A Direct Proof of Cauchy-Kovalevsky's Theorem}

\subsection{FORMULATION OF THE PROBLEM}

The Cauchy-Kovalevsky theorem for partial differential equations in the complex field is usually proved by the method of majorants, which, for the problem under investigation, leads to a first order ordinary differential equation with separable variables, whose solutions are then proved to be majorants of the solutions of the given system of partial differential equations. All this is avoided in the following simple proof (No. (2.5) below), based on functional analysis.

Let $\checkmark$ denote the class of all functions $u$ of $t$ and $z=\left(z_{1}, \ldots, z_{v}\right)$, which are holomorphic in some complex neighborhood of the origin $(t=0, z=0$ ), and thus possess power series expansion $u(t, z)=\sum_{\ell} \sum_{m} u_{\ell m} t^{\ell} z_{1} m_{1} \ldots z_{v}{ }^{m_{v}}$, $m=\left(m_{1}, \ldots, m_{v}\right)$, which is convergent in some neighborhood of the origin.

For the sake of simplicity we limit ourselves to the linear Cauchy problem, and we know that it is not restrictive (see I. G. Petrovsky [32], pp. 1617) to formulate the linear Cauchy problem as follows: Given elements a ijh' $b_{i j}, c_{i} \in \mathcal{S}$, determine elements $u_{i} \in \mathcal{S}$ such that

$$
\begin{aligned}
& u_{i t}=\sum_{j=1}^{n} \sum_{h=1}^{v} a_{i j h} u_{j z_{h}}+\sum_{j=1}^{n} b_{i j} u_{j}+c_{i}, \\
& u_{i}\left(0, z_{1}, \ldots, z_{v}\right)=0, \quad i=1, \ldots, n,
\end{aligned}
$$

in some complex neighborhood of the origin, and where $u_{i t}, u_{j z_{h}}$ denote partial derivatives with respect to $t$ and $z_{h}, h=1, \ldots, v$. If $U(t, z)=\left(u_{1}, \ldots, u_{v}\right)$, 
and $U_{z}=\left[u_{i z_{h}}, i=1, \ldots, n, h=1, \ldots, v\right]$, then $(1)$ takes the form

$$
U_{t}=F\left(t, z, U, U_{z}\right), U(0, z)=0 \text {. }
$$

The solution $U=\left(u_{1}, \ldots, u_{n}\right) \in \mathcal{S}^{\mathrm{n}}$ of problem (l) is uniquely determined by the usual argument: If $\sum_{\ell} \sum_{m} u_{i \ell m} t^{\ell} z_{1} m_{1} \ldots z_{v}{ }^{m_{v}}, i=1, \ldots, n$, are power series satisfying formually $U_{t}=F\left(t, z, U, U_{z}\right)$, and the coefficients $u_{\text {iom }}$ are known, then all remaining coefficients can be determined by induction. Indeed, each power $t^{\ell} z_{1}^{m_{1}} \ldots z_{v}^{m_{v}}$ appears in the first member of (I) with coefficients $(\ell+1) u_{i, l+1, m}$ and in the second member with coefficients $E_{i l m}$ which are finite linear combinations of coefficients $u_{j \lambda, s}, s=\left(s_{1}, \ldots, s_{v}\right), j=1, \ldots, n$, with $0 \leq \lambda \leq \ell$. For a solution $U \in \mathcal{S}^{\mathrm{n}}$ of problem (1) all $u_{\text {iom }}$ are zero.

A proof of the following theorem will be given in (2.5).

$$
\begin{aligned}
& \text { (2.i) (Cauchy-Kovalevsky). If all } a_{i j h}, b_{i j}, c_{i} \text { are elements of } \mathcal{S}, \\
& \text { then there is a unique element } u \in \mathcal{S} \text { satisfying (l) in a } \\
& \text { complex neighborhood of the origin } t=0, z=0 .
\end{aligned}
$$

\subsection{BANACH SPACES OF HOLOMORPHIC FUNCTIONS}

For $\delta>0,0<\alpha \leq 1$, and $k \geq 0$ integer, let $S_{k}=S_{\delta}$ be the class of all elements $u \in \mathcal{S}$ with coefficients $u_{\ell m}$ for which

$$
\|u\|_{\delta \alpha k}=\sup _{\ell, m}\left[\left|u_{\ell m}\right| \ell ! m_{1} ! \ldots m_{v} ! k !\left(\left(\ell+m_{1}+\ldots+m_{v}+k\right) !\right)^{-1} \alpha^{\ell} \delta^{\ell+m_{1}+\ldots+m_{v}}\right]<\infty
$$

Then $S_{k}$ is a Banach space with norm $\|u\|_{k}=\|u\|_{\delta \alpha k}$ Actually, we shall need below only $S_{0}$ and $S_{1}$. 
If $u \in S_{k}$, then $u_{t}, u_{z_{h}} \in S_{k+1}$ with $\left\|u_{t}\right\|_{k+1} \leq(k+1)(\alpha \delta)^{-1}\|u\|_{k}$, $\left\|u_{z_{h}}\right\|_{k+1} \leq(k+1) \delta^{-1}\|u\|_{k}, h=1, \ldots, v$. Conversely, if $v \in S_{k+1}$ and $u(t, z)=$ $\int_{0}^{t} \mathrm{v}(\tau, \mathrm{z}) \mathrm{d} \tau$ (formal integration), then $\mathrm{u} \in \mathrm{S}_{\mathrm{k}}$ and $\|\mathrm{u}\|_{\mathrm{k}}=(\mathrm{k}+1)^{-1}(\alpha \delta)\|\mathrm{v}\|_{\mathrm{k}+1}$. If $0<\delta<\sigma, u \in S_{\delta \alpha k}, v \in S_{\sigma \alpha k}$, then $u v \in S_{\delta \alpha k}$ and $\|u v\|_{\delta \alpha k} \leq(1-\delta / \sigma)^{-k-1}$ $\|\mathrm{u}\|_{\delta \alpha k}\|v\|_{\sigma \alpha k} \cdot$

Note that $S_{\delta \alpha, k+1} \supset S_{\delta \alpha k}$, and that $u \in S_{\delta \alpha k}$ implies $\|u\|_{\delta \alpha k} \geq\|u\|_{\delta \alpha, k+1} \cdot$ Also, for $0<\alpha<\beta \leq 1$, we have $S_{\delta \alpha k} \supset S_{\delta \beta k}$, and $\|u\|_{\delta \alpha k} \leq\|u\|_{\delta \beta k}$.

For $U(t, z)=\left(u_{1}, \ldots, u_{n}\right) \in\left(S_{\delta \alpha k}\right)^{n}$, that is, each $u_{i} \in S_{\delta \alpha k}$, we take $\|U\|_{\delta \alpha k}=\left(\sum_{i=1}^{n}\left\|u_{i}\right\|_{\delta \alpha k}^{2}\right)^{I / 2}$. Note that if $u \in S_{k}$ then $u$ has the majorant $\|u\|_{k}\left(1-\delta^{-1}\left(t / \alpha+z_{1}+\ldots+z_{v}\right)\right)^{-k-1}$, the same kind of majorant used in the classical proof of Cauchy-Kovalevsky theorem (see I. G. Petrovsky [32], pp. 18-24).

\subsection{A PRIORI ESTIMATE}

Let $\sigma>0$ be such that $a_{i j h}, b_{i j}, c_{i} \in S_{\sigma_{10}}$, and take $A=\operatorname{Max}\left[\left\|a_{i j h}\right\|_{\sigma_{1} 0}\right.$, $\left.\left\|b_{i j}\right\|_{\sigma 10}\right]$. Then, for every $0<\delta \leq \sigma, 0<\alpha \leq 1$, we have $\left\|a_{i j h}\right\|_{\delta \alpha \sigma^{\prime}}\left\|b_{i j}\right\|_{\delta \alpha 0} \leq A$. If $C$ denotes the $n$-vector $C=\left[c_{i}, i=1, \ldots, n\right]$, then we have also $\|C\|_{\delta \alpha 0} \leq\|C\|_{\sigma \alpha 0^{\circ}}$ Let us choose $\delta$ and $\alpha$ so that $0<\delta \leq 2^{-1} \sigma, 0<\alpha \leq 1,4 \mathrm{An}^{2}(\delta+v) \alpha<1$. Let us prove that for any solution $U \in S_{0}^{n}=\left(S_{\delta \alpha 0}\right)^{n}$ of $(1)$, if any exists, we have

$$
\|U\|_{0} \leq \alpha \delta\left(1-4 n^{2} A(v+\delta) \alpha\right)^{-1}\|C\|_{0}=\mu\|C\|_{0}
$$

Indeed, if $U \in S_{0}^{n}$, then $U_{t}, U_{z_{n}} \in S_{I}^{n}=\left(S_{\delta \alpha I}\right)^{n}, h=1, \ldots, v$. On the other hand, $U(t, z)=\int_{0}^{t} U_{\tau}(\tau, z) d \tau$, hence $\|U\|_{0}=\alpha \delta\left\|U_{t}\right\|_{1}$. Thus,

$$
\begin{aligned}
& \|U\|_{0}=\alpha \delta\left\|U_{t}\right\|_{1}=\alpha \delta\left\|F\left(t, z, U, U_{z}\right)\right\|_{1} \\
& \leq \alpha \delta\left[n^{2} v(1-\delta / \sigma)^{-2} A\left(\operatorname{Max}\left\|U_{z_{1}}\right\|+n^{2}(1-\delta / \sigma)^{-2} A\|\|_{1}+\|C\|_{1}\right],\right.
\end{aligned}
$$


where $1-\delta / \sigma \geq \mid 2^{-1},\left\|U_{z_{h}}\right\|_{1} \leq \delta^{-1}\|\|_{0},\|U\|_{1} \leq\|U\|_{0},\|\mathrm{c}\|_{1} \leq\|\mathrm{C}\|_{0}$. We obtain

$$
\|U\|_{0} \leq \alpha \delta\left[4 n^{2} v A \delta^{-1}\|U\|_{0}+4 n^{2} A\|U\|_{0}+\|C\|_{0}\right]
$$

which yields (2).

Note that relation (2) yields another proof that there can be at most one solution $U \in \mathcal{S}$ to problem (1). Indeed, any two solutions $U_{1}, U_{2} \in \mathcal{S}_{\text {must belong }}$ to the same space $S_{\delta \alpha 0}$ for $\delta, \alpha$ chosen as above, and then $U_{1}-U_{2}$ is a solution of (1) with $\mathrm{C}=0$. By (2) we have then $\left\|U_{1}-U_{2}\right\|_{0}=0$, or $U_{1}=U_{2}$.

\subsection{PROOF OF THE EXISTENCE OF A SOLUTION TO PROBLEM (1)}

Let us denote by $\mathrm{H}$ the operator of integration with respect to $t$ from 0 to t already considered in No. 3. Then, problem (1) is equivalent to

$$
U(t, z)=H F\left(t, z, U, U_{z}\right)
$$

Thus, if $\mathrm{T}: \mathcal{S}^{\mathrm{n}} \rightarrow \mathcal{S}^{\mathrm{n}}$ denotes the operator defined by,

$$
\mathrm{V}=\mathrm{TU}=\mathrm{HF}\left(\mathrm{t}, \mathrm{z}, \mathrm{U}, \mathrm{U}_{\mathrm{z}}\right)
$$

all we have to do is to find the fixed points of $T$.

Let $\sigma, A, \delta, \alpha$ be chosen as in No. 4 so that $k=4 n^{2} A(v+\delta) \alpha<1$. Note that, if $U \in S_{0}^{n}$, then $U_{z} \in S_{l}^{n}$, and $F\left(t, z, U, U_{z}\right) \in S_{1}^{n}$. Then, $H F \in S_{0}^{n}$, and $T$ is actually a map $T: S_{0}^{n} \rightarrow S_{0}^{n}$. If $U_{1}, U_{2} \in S_{0}^{n}$, then

$$
\begin{aligned}
\left\|\mathrm{TU} U_{1}-\mathrm{TU}_{2}\right\|_{0} & =\alpha \delta\left\|\mathrm{TU} U_{1}-\mathrm{TU}_{2}\right\|_{1}= \\
& =\alpha \delta\left\|F\left(t, z, U_{1}, U_{1 Z}\right)-F\left(t, z, U_{2}, U_{2 Z}\right)\right\|_{1}^{-}
\end{aligned}
$$




$$
\begin{aligned}
& \leq \alpha \delta\left[n^{2} v(1-\delta / \sigma)^{-2} A\left(\max \left\|U_{1 z_{h}}-U_{2 z_{h}}\right\|\right)\right. \\
& \left.+n^{2}(1-\delta / \sigma)^{-2} A\left\|U_{1}-U_{2}\right\|_{1}\right] \\
& \leq 4 n^{2} A(v+\delta) \alpha\left\|U_{1}-U_{2}\right\|_{0}=k\left\|U_{1}-U_{2}\right\|_{0} .
\end{aligned}
$$

Since $k<1, T: S_{0}^{n} \rightarrow S_{0}^{n}$ is a contraction in the norm \|\|$_{0}$ of $S_{0}^{n}$, and possesses, therefore, a unique fixed point $U \in S_{0}^{n} \subset S^{n}$.

\subsection{PROJECTION OPERATORS}

For fixed integers $N \geq 1, M \geq 1$, let us denote by $P_{N}^{t}, P_{M}^{z}: \mathcal{S} \rightarrow$, the projection operators defined by

$$
\begin{aligned}
& P_{N}^{t}\left(\sum_{l} \sum_{m} u_{l m} t^{l} z_{1}^{m_{1}} \cdots z_{v}^{m_{v}}\right)=\sum_{l=0}^{N} \sum_{m} u_{l m} t^{l} z_{1}^{m_{1}} \cdots z_{v}^{m_{v}} \\
& P_{M}^{z}\left(\sum_{l} \sum_{m} u_{l m} t^{l} z_{1}^{m_{1}} \cdots z_{v}^{m_{v}}\right)=\sum_{l} \sum_{m_{1}=0}^{M} \cdots \sum_{m_{v}=0}^{M} u_{l m} t^{l} z_{1}^{m_{1}} \cdots z_{v}^{m_{v}},
\end{aligned}
$$

so that we have also $P_{N}^{t}, P_{M}^{z}: S_{k} \rightarrow S_{k}$. For fixed $N$ and $M$, both $P_{N k}^{t} S_{k}$ and $P_{M}^{z} S_{k}$ are subspaces of $\mathrm{S}_{\mathrm{k}}$, actually Banach spaces in the same norm $\|\mathrm{u}\|_{\mathrm{k}}$ of $\mathrm{S}_{\mathrm{k}}$.

For any fixed $M \geq I$ we may consider the Cauchy problem analogous to ( $I$ ):

$$
U_{t}=P_{M}^{z} F\left(t, z, U, U_{z}\right), U(0, z)=0 \text {, }
$$

for which we seek solutions $U \in\left(P_{M}^{Z} S_{0}\right)^{n}$. The argument of No. 2 leading to the uniqueness of a solution $U$, if any exists, repeats in the present situation.

The same holds for the argument of No. 4 leading to an a priori estimate. Thus, and solution $U \in\left(\mathrm{P}_{\mathrm{m}}^{\mathrm{z}} \mathrm{S}_{0}\right)^{\circ}$ of problem (3), if any exists, is uniquely determined and satisfies relation (2). 
Analogously, for fixed $N, M \geq I$ we may consider the Cauchy problem analogous to (1) and (3):

$$
U_{t}=P_{N-1}^{t} P_{M}^{z} F\left(t, z, U, U_{z}\right), U(0, z)=0
$$

for which we seek solutions $U \in\left(P_{N}^{t} P_{M}^{z} S_{0}\right)^{n}$ (polynomials in $t$ and $\left.z\right)$. Both the argument leading to the uniqueness of a solution, and the argument leading to the a priori estimate hold in the present situation. But now $U$ is a polynomial, and thus we conclude that a polynomial solution $U \in\left(P_{N}^{t} P_{M}^{z} S_{0}\right)^{n}$ of problem (4) exists and $U$ satisfies (2).

\subsection{AN ALTERNATE PROOF OF THE EXISTENCE OF A SOLUTION TO PROBLEM (1)}

For every $M=1,2, \ldots$, let us take $N=M$ in problem $(4)$, and let $U^{M}(t, z)$ be the polynomials so obtained, $U^{M} \in\left(P_{N}^{t} P_{M}^{z} S_{0}\right)^{n}$. Let $u_{i l m}^{M}$ be the coefficient of the polynomials $U^{M}$. All these $U^{M}$ are elements of the unique Banach space $S_{0}^{n}$, and satisfy the a priori estimate $\left\|U^{M}\right\|_{0} \leq \mu$, or (2), as proved. Let $u_{i l m}$ be the coefficients of the formal power series expansions corresponding to problem (1), as mentioned in No. 2. Then for every fixed $l$ and $m=\left(m_{1}, \ldots, m_{v}\right)$ and all $M$ large enough, we have $u_{i l m}^{M}=u_{i l m}$. Hence, also $u_{i l m}$ are the coefficients of an element $U \in S_{0}^{n}$ and $\|U\|_{0} \leq \mu$. Thus $U \in S_{0}^{n} \subset \mathcal{S}$, and $U$ is a solution to problem (I).

\subsection{ANOTHER PROOF}

Let $g(t, z)=\left(g_{1}, \ldots, g_{n}\right)$ be any $n$-vector polynomial, or element $g \in\left(P_{N}^{t} P_{M}^{z} S_{0}\right)^{n}$, with $g(0, z)=0$, and let $g_{i l m}, 0 \leq l \leq N, 0 \leq m_{h} \leq M$, denote the coefficients of $g_{i}\left(\right.$ ali $g_{i o m}$, and all $g_{i l m}$ with $\ell>N$ or $m_{h}>M$, being zero). 
For every $U(t, z)=\left(u_{1}, \ldots, u_{n}\right) \in S_{0}^{n}$, let $u_{i l m}$ denote the coefficients of the power series of $u_{i}$, and let $\mathrm{H}: \mathrm{S}_{0} \rightarrow \mathrm{S}_{0}$ denote the operation of integration with respect to $t$ as in $N o .3$, or $H U=V(t, z)=\left(V_{1}, \ldots, V_{n}\right)$ with

$$
v_{i}(t, z)=\sum_{\ell=N}^{\infty} \sum_{m} u_{\ell m}(\ell+1)^{-1} t^{\ell+1} z_{1} m_{1} \ldots z_{v}^{m_{v}}, \quad i=1, \ldots, n .
$$

Then, $(\partial / \partial t) H U=U-P_{N-1}^{t} U,\|H U\|_{0} \leq(N+1)^{-1}\|U\|_{0}$, and also $H:\left(P_{M_{0}}^{z}\right)^{n} \rightarrow$

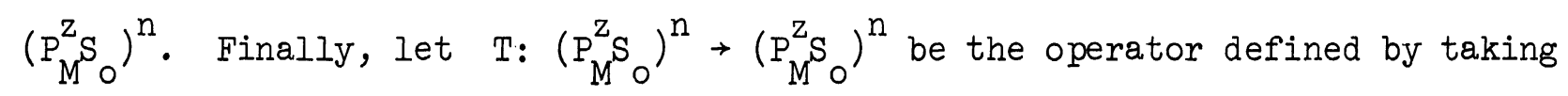

$$
V=T U=g+\left(I-P_{M}^{z}\right) F\left(t, z, U, U_{z}\right)
$$

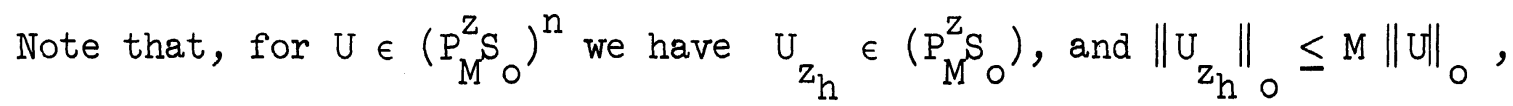
$h=1, \ldots, v$. Then, for $U, V \in\left(P_{M_{0}^{S}}^{z_{0}}\right)^{n}$ we have also, as in No. 4,

$$
\|T U-T V\|_{0} \leq(N+1)^{-1}\left(2 A M n^{2} v+2 A n^{2}\right)\|U-V\|_{0}
$$

By choosing $N=N(M)$ sufficiently large, $T$ is a contraction. The fixed point $U=T U \in\left(P_{M}^{Z} S_{0}\right)^{n}$ is an element $U=T U=\tau$ g which depends on $g$, and which satisfies $U=g+H P_{M}^{Z} F\left(t, z, U, U_{z}\right)$ with $u_{i l m}=g_{i l m}$ for $0 \leq l \leq N$. By differentiation we obtain

$$
U_{t}=P_{M}^{z} F\left(t, z, U, U_{z}\right)+\Delta,
$$

where $\Delta=g_{t}-P_{N-1}^{t} P_{M}^{Z} F$. Thus, $U=T U=\tau g$ is a solution of (4) if and only if the determining (or bifurcation) equation

$$
g_{t}=P_{N-1}^{t} F\left(t, z, U, U_{z}\right)
$$

is satisfied. Here $u_{i l m}=g_{i l m}$ for all $\ell \leq N$ and $g_{i o m}=0$, and the determination 
of a polynomial $g$ satisfying (6) is a problem similar to (4). Thus g can be uniquely determined so as to satisfy $(6)$, and correspondingly $U=T U=\tau_{g}$ satisfies problem (4). If we denote by $U^{M} \in\left(P_{M}^{Z} S_{0}\right)^{n}$ the element so determined, and we take $M=1,2, \ldots$, we have a sequence $\left[U^{M}\right]$ as in No. (2.6) and a solution U of the original problem (I) can be derived. 


\subsection{STATEMENT OF THE PROBLEM}

The proof and the remaining considerations of No. 2 extend to a more general statement (Hormander [28], Th. 5.1., p. 116), from which Hormander deduces as corollaries the Cauchy-Kovalevsky theorem, a theorem by Darboux, .. Goursat, and Bendon, and a number of other statements ([28], pp. 118-126). For the sake of simplicity we limit ourselves to partial differential equations with one unknown function. The extension to systems is easy.

Let $\checkmark$ denote the class of all holomorphic functions $u(z), z=\left(z_{1}, \ldots, z_{v}\right)$, which are holomorphic in some complex neighborhood of the origin $z=0$, and thus possesses power series expansion $\sum_{m} u_{m} z^{m}$, or $\sum_{m} u_{m} z_{l} m_{1} \ldots z_{v}{ }_{v}, m=$ $\left(m_{1}, \ldots, m_{v}\right),|m|=m_{1}+\ldots+m_{v}$. Hormander's problem is as follows:

Given any multiindex $\beta=\left(\beta_{1}, \ldots, \beta_{\nu}\right),|\beta|>0$, and elements $f, a, \varphi \in \varsigma$, $0 \leq|\alpha| \leq|\beta|$, determine an element $u \in \mathcal{S}$ such that in a neighborhood of $z=0$ we have

$$
\begin{gathered}
D^{\beta} u=|\alpha| \leq|\beta| a^{\alpha}(z) D^{\alpha} u+f(z) \text {, and } \\
D_{j}^{k}(u-\varphi)=0 \text { when } z_{j}=0 \text { if } 0 \leq k<\beta_{j}, j=1, \ldots, v .
\end{gathered}
$$

A proof of the following theorem will be given in No. 3.5 below. As usual $D^{\beta} u$ denotes $\partial^{|\beta|} u / \partial z_{1} \beta_{1} \ldots \partial z_{v}^{\beta}$. 
(3.1.i) (Hormander, [28] Th.5.1.1, p. 116). If $\sum\left|a^{\alpha}(0)\right|$ is smaller $|\alpha|=|\beta|$

than a positive number depending only on $|\beta|$, then problem (1),

(2) has one and only one solution $u \in \mathcal{S}$.

\subsection{THE CLASSICAL UNIQUENESS ARGUMENT}

The substitution $u=v+\varphi$ reduces the proof to the case $\varphi=0$ so we may assume that $\varphi=0$ from the beginning. Thus, we replace (2) by

$$
D_{j}^{k} u=0 \text { when } z_{j}=0 \text { if } 0 \leq k<\beta_{j}, j=1, \ldots, v \text {. }
$$

If $\alpha, \beta$ are any two multiindices $\alpha=\left(\alpha_{1}, \ldots, \alpha_{v}\right), \beta=\left(\beta_{1}, \ldots, \beta_{v}\right)$ we shall say that $\alpha<\beta$ [or $\alpha \leq \beta$ ] provided $\alpha_{i}<\beta_{i}\left[\right.$ or $\alpha_{i} \leq \beta_{i}$ ] for all $i=1, \ldots, v$. If $\alpha \leq \beta$ we shall write as usual

$$
\left(\begin{array}{l}
\beta \\
\alpha
\end{array}\right)=\left(\begin{array}{l}
\beta_{1} \\
\alpha_{1}
\end{array}\right) \ldots\left(\begin{array}{l}
\beta_{v} \\
\alpha_{v}
\end{array}\right) \text { with }\left(\begin{array}{c}
\beta_{i} \\
\alpha_{i}
\end{array}\right)=\frac{\beta_{i} !}{\alpha_{i} !\left(\beta_{i}-\alpha_{i}\right) !} .
$$

Given $\beta=\left(\beta_{1}, \ldots, \beta_{\nu}\right)$ and any integer $\lambda|\beta| \beta \mid$, we shall denote by $N_{\lambda \beta}$ the number of integral solutions of the equations $|l|=\lambda, l \geq \beta$, or $l_{1}+\ldots+l_{v}=\lambda$, $\ell_{i} \geq \beta_{i}, i=1, \ldots, v$. Also, we shall denote by $\mu_{\beta}$ the number of solutions of the equations $|\alpha|=|\beta|$, or $\alpha_{1}+\ldots+\alpha_{v}=\beta_{1}+\ldots+\beta_{v}$.

The solution $u \in \mathcal{S}$ of problem (1-3) is uniquely determined. This can be proved by a simple modification of the usual argument for the Cauchy-Kovalevsky theorem (see [5], p. 19). Indeed, if $u \in \mathcal{V}$ satisfies (1-3), and $d_{\ell}, A_{\alpha \ell}$, $\cdot l=\left(l_{1}, \ldots, l_{v}\right)$ denote the derivatives $a_{\ell}=\left.D^{\ell}\right|_{z=0}, A_{d}=\left.D^{l} a\right|_{z=0}$, then all derivatives $d_{l}$ with at least one $l_{i}<\beta_{i}$ are zero. In other words, $d_{l}=0$ for every $\ell \geq \beta$. For $\ell \geq \beta$, the derivatives $d_{l}$ can be obtained from Leibniz rule by differentiating ( 1 ) exactly $\ell-\beta$ lines, that is, applying the operator 
$D^{\ell-\beta}$ on both sides, and taking $z=0$. We obtain

$$
d_{\ell}=\sum_{|\alpha| \leq|\beta|} \sum_{\ell^{\prime} \leq \ell}\left(\begin{array}{l}
\ell \\
\ell^{\prime}
\end{array}\right) A_{\alpha, \ell-\ell^{\prime}} \ell_{\ell^{\prime}+\alpha-\beta} ;
$$

and in this sum we may well restrict to only those terms with $\ell^{\prime}+\alpha-\beta \geq \alpha$, or $\ell^{\prime} \geq \beta$. Note that, for any given $\ell \geq \beta$ we have in $(4) \ell^{\prime} \leq \ell, \ell \geq \beta, \ell^{\prime} \geq \beta$, $|\alpha| \leq|\beta|$, and finally

$$
\left|\ell^{\prime}+\alpha-\beta\right|=\left|\left(\ell^{\prime}-\beta\right)+\alpha\right|=\left|\ell^{\prime}-\beta\right|+|\alpha|=\left|\ell^{\prime}\right|-|\beta|+|\alpha| \leq\left|\ell^{\prime}\right| \leq|\ell| \text {. }
$$

Thus, for any given $\lambda \geq 0, \lambda \geq|\beta|,|\ell|=\lambda$, we may have in (4) $\left|\ell^{\prime}+\alpha-\beta\right|=\lambda$ if and only if $\left|\ell^{\prime}\right|=|\ell|,|\beta|=|\alpha|$. In other words, the $N_{\lambda}$ derivatives $d_{\ell}$ with $|l|=\lambda, l \geq \beta$, satisfy the system of $\mathrm{N}_{\lambda}$ equations

$$
d_{\ell}-\sum_{|\alpha|=|\beta|} A_{\alpha} d_{\ell+\alpha-\beta}=E_{\ell},|\ell|=\lambda, \quad \ell \geq \beta
$$

where $E_{l}$ denote finite linear combinations of derivatives $d_{s}$ with $|s|<\lambda$, and where $A_{\alpha 0}=a^{\alpha}(0)$. In each equation (5) there appear, therefore, besides the unknown $d_{l}$ with coefficient one, at most $\mu_{\beta}$ other unknowns $d_{\ell+\alpha-\beta}$ with $l+\alpha-\beta=$ $|\lambda|=|\ell|$, with coefficients $A_{\alpha 0}$ whose sum of the absolute values is $\leq \sum_{|\alpha|=|\beta|}$ $\left|a^{\alpha}(0)\right|$. Thus, if this sum is $<1$, the $N_{\lambda}$ equations (5) have a unique solution ([22] p. 29).

\subsection{BANACH SPACES OF HOLOMORPHIC FUNCTIONS}

For $\delta>0$ and $k \geq 0$ integer, let $s_{\delta k}$ be the class of all elements $u \in \mathcal{S}$, $u=\sum_{m} u_{m} z_{1} \ldots z_{v} m_{v}, m=\left(m_{1}, \ldots, m_{v}\right)$, for which

$$
\|u\|_{\delta k}=\sup _{m}\left[\left|u_{m}\right| m_{1} ! \ldots m_{v}: k !\left(\left(m_{1}+\ldots+m_{v}+k\right) !\right)^{-1} \delta^{m_{1}+\ldots+m_{v}}\right]<\infty .
$$


Then $S_{\delta k}$ is a Banach space with norm $\|u\|_{\delta k^{*}}$ Note that $S_{\delta k} \subset S_{\delta, k+1}$ and that for $u \in S_{\delta k}$ we have $\|u\|_{\delta k} \geq\|u\|_{\delta, k+1}$. Also, $s_{\delta o} \subset S_{\delta l} \subset \ldots \subset s_{\delta k} \subset \ldots \subset \mathcal{S}$, for $u \in S_{\delta \circ}$ we have $\|u\|_{\delta 0} \geq\|u\|_{\delta I} \geq \ldots \geq\left|u_{0}\right|$. For any given element $u \in S_{\sigma o}$ we can take $\delta, 0<\delta \leq \sigma$, so small that $\|u\|_{\delta 0}$ is a close as we want to $\left|u_{0}\right|$. If $u \in S_{\delta k}$ then each derivative $D_{x_{i}} u \in S_{\delta, k+1}$ with $\left\|D_{x_{i}} u\right\|_{\delta, k+1} \leq(k+1) \delta^{-1}\|u\|_{\delta k}$. Conversely, if $v \in S_{\delta, k+1}$ and $u(z)=D_{x_{i}}^{-1} v$ (formal integration), then $u \in S_{\delta k}$ and $\|u\|_{\delta k}=(k+1)^{-1} \delta\|v\|_{\delta, k+1}$. These are immediate consequences of the definitions. As a consequence, for every multiindex $\alpha=\left(\alpha_{1}, \ldots, \alpha_{v}\right)$ and $u \in S_{\delta k}$, we have $D^{\alpha} u \in S_{\delta, k+|\alpha|}$ and $\left\|D^{\alpha} u\right\|_{\delta_{1} k+|\alpha|} \leq(k+1) \ldots(k+|\alpha|) \delta^{-|\alpha|}\|u\|_{\delta k^{\cdot}}$ Conversely, if $\mathrm{v} \in \mathrm{S}_{\delta, \mathrm{k}+|\alpha|}$ and $\mathrm{u}=\mathrm{D}^{-\alpha} \mathrm{v}$ (formal integration), then $\mathrm{u} \in \mathrm{S}_{\delta \mathrm{k}}$ and $\|\mathrm{u}\|_{\delta \mathrm{k}}=$ $(\mathrm{k}+1)^{-1} \ldots(\mathrm{k}+|\alpha|)^{-1} \delta|\alpha|\|\mathrm{u}\|_{\delta, \mathrm{k}+|\alpha|}$.

Note that, if $0<\delta<\sigma, u \in S_{\delta k}, v \in S_{\sigma k}$, then $u v \in S_{\delta k}$ and $\|u v\|_{\delta k}$ $\leq(1-\delta / \sigma)^{-k-1}\|u\|_{\delta k}\|v\|_{\sigma k}$. Also note that, if $u \in S_{\delta k}$, then

$$
\left|u_{m}\right| \leq\|u\|_{\delta k}\left(m_{1}+\ldots+m_{v}+k\right) !\left(m_{1} ! \ldots m_{v} ! k !\right)^{-1} \delta^{-m_{1}-\ldots-m_{v}}
$$

that is, $u(z)$ has the majorant

$$
\|u\|_{\delta k} \sum_{m}\left(m_{1}+\ldots+m_{v}+k\right) !\left(m_{1} \ldots \ldots m_{v} ! k !\right)^{-1} z_{1}^{m_{1}} \ldots z_{v}^{m_{1}} \delta^{-m_{1}-\ldots-m_{v}}
$$

or

$$
\|u\|_{\delta k}\left(1-\left(z_{1}+\ldots+z_{v}\right) \delta^{-1}\right)^{-k-1}
$$

Conversely, if $u(z)$ has a majorant $\mu\left(1-\left(z_{1}+\ldots+z_{v}\right) \delta^{-1}\right)^{-k-1}$, then $u \in S_{\delta k}$ and $\|\mathrm{u}\|_{\delta \mathrm{k}} \leq \mu$ 


\subsection{A PRIORI ESTIMATE}

We prove here an a priori estimate for the solutions $u \in \checkmark$ of problem (1-3) above (that is, for problem (5.1.1) of [28], p. 116).

(3.4.i) Given $\beta$, there is a number $\tau=\tau(\beta)>0$ and, for all a $a^{\alpha} \mathcal{V}$

$|\alpha| \leq|\beta|$ with $|\alpha|=|\beta| a^{\alpha}(0) \mid \leq \tau$, there is another number $\delta_{0}>0$ such that, for any $f \in S_{\delta 0}, 0<\delta \leq \delta$, and solution $u \in \mathcal{G}$ of (1-3) (if any) with $u \in S_{\delta o}$, we have also

$$
\|u\|_{\delta_{0}} \leq 2(|\beta| !)^{-1} \delta^{|\beta|}\|f\|_{\delta_{0}}
$$

Proof. Let $\tau=2^{-4-|\beta|}$. Given $a^{\alpha} \in \mathcal{S},|\alpha| \leq|\beta|$, let $\sigma>0$ be such that $a^{\alpha} \in S_{\sigma 0^{\prime}},|\alpha| \leq|\beta|$, and let $A=\operatorname{Max}\left[\| a_{\sigma 0^{\prime}},|\alpha|<|\beta|\right]$. Then, for any $r$ integer we have $\left\|a^{\alpha}\right\|_{\sigma r} \leq\|a\|_{\sigma o} \leq A$ for all $\alpha$ with $|\alpha|<|\beta|$. If $|\alpha|_{|\beta|}\left|a^{\alpha}(0)\right| \leq \tau$, then we certainly have

$$
2^{|\beta|+1}|\alpha| \sum_{|\beta|}\left|a^{\alpha}(0)\right|<2^{-3}
$$

Let $\delta_{0}$ be so chosen that $0<\delta_{0} \leq 2^{-1} \sigma$,

$$
2^{|\beta|+1} A|\alpha|<|\beta| \delta_{0}^{|\beta|-|\alpha|}<2^{-3},
$$

and such that

$$
\sum_{|\alpha|=|\beta|}\left\|a^{\alpha}\right\|_{\delta 0}<\sum_{|\alpha|=|\beta|}\left|a^{\alpha}(0)\right|+2^{-p-4}
$$

for every $\delta, 0<\delta \leq \delta_{0}$. Then

$$
\begin{aligned}
k_{0} & =2^{|\beta|+1} A \sum_{|\alpha|<|\beta|} \delta_{0}^{|\beta|-|\alpha|}+2^{|\beta|+1}\left(\sum_{|\alpha|=|\beta|}\left|a^{\alpha}(0)\right|+2^{-|\beta|-4}\right) \\
& <2^{-3}+2^{-3}+2^{-3}<2^{-1} .
\end{aligned}
$$


Thus, for $0<\delta \leq \delta$, we have $1-\delta / \sigma \geq 2^{-1}$, and if $k(\delta)$ is the number defined as in (9) with $\delta$ replacing $\delta_{0}$ and $\mu(\delta)=1-k(\delta)$, we have $0<k(\delta) \leq k_{0}<2^{-1}$, $1>\mu(\delta)>1-2^{-1}=2^{-1}$.

If $f \in S_{\delta o}$ and $u \in \mathcal{S}, u \in S_{\delta 0}$, is any solution of $(1-3)$, then $D^{\beta} u \in S_{\delta,|\beta|}$, and $u=D^{-\beta}\left(D^{\beta} u\right.$ ) (formal integration as in No. (3.3)). Then from (1) and the remarks made in No. $(3.3)$,

$$
\begin{aligned}
& \|u\|_{\delta 0}=(|\beta| !)^{-1} \delta^{|\beta|}\left\|D^{\beta} u\right\|_{\delta,|\beta|}= \\
& =(|\beta| !)^{-1} \delta|\beta|\left\|\sum_{|\alpha| \leq|\beta|} a^{\alpha} D^{\alpha} u\right\|_{\delta,|\beta|} \\
& \leq(|\beta| !)^{-1}|\beta|\left[(1-\delta / \sigma)^{-|\beta|-1} \sum_{|\alpha| \leq|\beta|}\left\|a^{\alpha}\right\|_{\sigma,|\beta|}\left\|D^{\alpha} u\right\|_{\delta,|\beta|}+\|f\|_{\delta,|\beta|}\right] \\
& \leq(|\beta|:)^{-1} \delta{ }^{|\beta|}\left[2^{|\beta|+1} A \sum_{|\alpha|<|\beta|}(|\beta|-|\alpha|+1) \ldots(|\beta|) \delta^{-|\alpha|}\|u\|_{\delta,|\beta|-|\alpha|}\right. \\
& \left.+2^{|\beta|+1}(|\beta| !) \delta^{-|\beta|}\left(\sum_{|\alpha|=|\beta|}\left|a^{\alpha}(0)\right|+2^{-\beta-4}\right)\|u\|_{\delta 0}+\|f\|_{\delta 0}\right] \\
& \leq 2^{|\beta|+1} A \sum_{|\alpha|<|\beta|} \delta^{|\beta|-|\alpha|}+2^{|\beta|+1}\left(\sum_{|\alpha|=|\beta|}\left|a^{\alpha}(0)\right|+2^{-\beta-4}\right)\|u\|_{\delta 0}+(|\beta|)^{-1} \\
& { }_{\delta}|\beta|\|f\|_{\delta 0} \text {, } \\
& \leq \mathrm{k}(\delta)\|u\|_{\delta 0}+(|\beta| !)^{-1} \delta|\beta|\|f\|_{\delta 0},
\end{aligned}
$$

or

$$
\|u\|_{\delta 0} \leq(\mu(\delta))^{-1}(|\beta|:)^{-1} \delta|\beta|\|f\|_{\delta 0}
$$

where $\mu(\delta)=1-k(\delta) \geq \mu_{0}>2^{-1}$, and (8) is thereby proved. 
3.5 PROOF OF THE EXISTENCE OF A SOLUTION TO PROBLEM (1-3)

Let us denote by $\mathrm{H}$ the operation of integration $\mathrm{H}=\mathrm{D}^{-\beta}$ already considered in Nos. (3.3) and (3.4). Then, problem (1-3) is equivalent to

$$
u(z)=H\left(\sum_{|\alpha| \leq|\beta|} a^{\alpha} D^{\alpha} u+f(z)\right)
$$

If $\mathrm{T}: \widetilde{S} \sim$ denotes the operator defined by

$$
v=T u=H\left(\sum_{|\alpha| \leq|\beta|} a^{\alpha} D^{\alpha} u+f(z)\right),
$$

all we have to do is to find fixed points of $\mathrm{T}$.

Let $\sigma, A, \delta$ be chosen as in No. (3.4), so that $0<k(\delta) \leq k_{0}<2^{-1}$.

Note that, if $u \in S_{\delta 0}$, then $D^{\alpha} u \in S_{\delta,|\alpha|}, 0 \leq|\alpha| \leq|\beta|$, and

$|\alpha| \leq|\beta|^{a} D^{\alpha} u \in S_{\delta,|\beta|}$. For $f \in S_{\delta, 0}$ we certainly have $H\left(|\alpha| \leq|\beta|^{a} D_{D}^{\alpha} u+f\right) \in S_{\delta 0^{\prime}}$, and $T$ is, therefore, a map $T: S_{\delta 0} \rightarrow S_{\delta 0}$. If $u_{1}, u_{2} \in S_{\delta 0^{\prime}}$, then

$$
\begin{aligned}
& \left\|T u_{1}-T u_{2}\right\|_{\delta 0}=\left\|H\left(\sum_{|\alpha| \leq|\beta|} a^{\alpha} D^{\alpha}\left(u_{1}-u_{2}\right)\right)\right\|_{\delta \circ} \\
& =(|\beta| !)^{-1} \delta|\beta|\left\|\sum_{|\alpha| \leq|\beta|} a^{\alpha} D^{\alpha}\left(u_{1}-u_{2}\right)\right\|_{\delta,|\beta|} \\
& \leq(|\beta|:)^{-1} \delta|\beta|(1-\delta / \sigma)^{-|\beta|-1} \sum_{|\alpha| \leq|\beta|}\left\|a_{\sigma,|\beta|^{\alpha}}\right\| D^{\alpha} u \|_{\delta,|\beta|} \\
& \leq(|\beta|:)^{-1} \delta|\beta|\left[2^{|\beta|+1} \sum_{|\alpha|<|\beta|}(|\beta|-|\alpha|-1) \ldots(|\beta|) \delta^{-|\alpha|}\left\|u_{1}-u_{2}\right\|_{\delta},|\beta|-|\alpha|{ }^{+}\right. \\
& \left.+2^{|\beta|+1}(|\beta|:) \delta^{-|\beta|}\left(\sum_{|\alpha|=|\beta|}\left|a^{\alpha}(0)\right|+2^{-|\beta|-4}\right)\right]\left\|u_{1}-u_{2}\right\|_{\delta \circ} \\
& \leq\left[2^{|\beta|+1} \sum_{|\alpha|<|\beta|} \delta|\beta|-|\alpha|+2^{|\beta|+1}\left(\sum_{|\alpha|=|\beta|}\left|a^{\alpha}(0)\right|+2^{-|\beta|-4}\right)\right]\left\|u_{1}-u_{2}\right\|_{\delta o} \\
& =k(\delta)|| u_{1}-\left.u_{2}\right|_{\delta 0},
\end{aligned}
$$


where $\mathrm{k}(\delta)<2^{-1}$. Thus, $\mathrm{T}: \mathrm{S}_{\delta 0} \rightarrow \mathrm{S}_{\delta 0}$ is a contraction in the norm $\|\mathrm{u}\|_{\delta 0}$ of $\mathrm{S}_{\delta 0}$, and possesses, therefore, a unique fixed point $u \in \mathrm{S}_{\delta 0^{\circ}}$. Theorem (3.1.i) is thereby proved.

Considerations analogous to the ones in Nos. (2.6-8) could be repeated here, and are omitted for the sake of brevity. 


\section{References}

\section{A. REFERENCES ON THE CESARI-HALE METHOD}

[1] H. R. Bailey and L. Cesari, Boundedness of solutions of linear differential systems with periodic coefficients. Archive Rat. Mech. and Anal. 1, 1958, 246-271.

[2] H. R. Bailey and R. A. Gambill, On stability of periodic solutions of weakly nonlinear differential equations. J. Math. Mech. 6, 1957, 655668 .

[3] C. Banfi, (a) Sulla determinazione delle soluzioni periodiche di equazioni non lineari periodiche. Boll. Unione Mat. Italiana, (4) 1, 1968, 608619.-(b) Su. un metodo di successive approssimazioni per lo studio delle soluzioni periodiche di sistemi debolmente nonlineari, Atti Acc. Sci. Torino, 100, 1968, 1065-1066.

[4] C. Banfi and G. Casadei, Calcolo di soluzioni periodiche di equazioni differenziali nonlineari periodiche. Congresso AICA, Napoli, Sept. 1968.

[5] L. Cesari, (a) Sulla stabilitá-delle soluzioni dei sistemi di equazioni differenziali lineari a coefficienti periodici. Mem. Accad. Italia (6) II, 1941, 633-695.-(b) Existence theorems for periodic solutions of nonlinear Lipschitzian differential equations and fixed-point theorems. Contributions to the Theory of Nonlinear Oscillations 5, Princeton 1960, pp. 115-172.-(c) Existence theorems for periodic solutions of nonlinear differential systems. Bol. Soc. Mat. Mexicana 5, 1960, 24-41.-(d) Asymptotic Behavior and Stability Problems in Ordinary Differential Equations, 2nd ed. Springer 1963.-(e) Functional analysis and Galerkin's method. Michigan Math. J. 11, 1964, 385-418.-(f) Functional analysis and periodic solutions of nonlinear differential equations. Contrib. Differential Equations, Wiley 1, 1963, 149-187.-(g) Periodic solutions of hyperbolic partial differential equations. Nonlinear Differential Equations and Nónlinear Mechanics. Academic Press, 1963, 33-57.(h) A criterion for the existence in a strip of periodic solutions of hyperbolic partial differential equations. Rend. Circ. Mat. Palermo (2) 14, 1965, 95-118.-(i) Existence in the large of periodic solutions of hyperbolic partial differential equations. Arch. Rat. Mech. Anal. 20, 1965, 170-190.-(j) Smoothness properties of periodic solutions in the large of nonlinear hyperbolic differential systems. Funkcialaj Etvacioj 9, 1966, 325-338.-(k) A nonlinear problem in potential theory. Michigan Math. J. 16, 1969, 3-20.-( $\ell$ ) Functional analysis and differential equations. SIAM Studies in Applied Mathematics 5, 1969, $143-$ 155. 
[6] L. Cesari and J. K. Hale, (a) Second order linear differential systems with periodic L-integrable coefficients, Riv. Mat. Univ. Parma, 2, 1954, 55-61; 6, 1955, 159.-(b) A new sufficient condition for periodic solutions of weakly nonlinear differential systems. Proc. Amer. Math. Soc. 8, 1957, 757-764.

[7] P. A. T. Christopher (a) A new class of subharmonic solutions to Duffing's equation. Co A Rep. 195, The College of Aeronautics. Cranfield, Bedford, England 1967.-(b) An extended class of subharmonic solutions to Duffing's equation. Co A. Rep. 199. Ibid. 1967.-(c) The response of a second order nonlinear system to a step-function disturbance. Co A Report 205, Ibid. 1969.

[8] J. Cronin, Fixed points and topological degree in nonlinear analysis. Amer. Math. Society, 1964.

[9] R. A. Gambill, (a) Stability criteria for linear differential systems with periodic coefficients. Riv. Mat. Univ. Parma, 5, 1954, 169-181. (b) Criteria for parametric instability for linear differential systems with periodic coefficients. Riv. Mat. Univ. Parma 6, 1955, 37-43.(c) A fundamental system of real solutions for linear differential systems with periodic coefficients. Riv. Mat. Univ. Parma 7, 1956, 311-319.

[10] R. A. Gambill and J. K. Hale, Subharmonic and ultraharmonic solutions for weakly nonlinear systems. J. Rat. Mech. Anal. 5, 1956, 353-398.

[11] A. Halanay, Differential Equations, Academic Press 1966 (particularly pp. 308-317).

[12] J. K. Hale, (a) Evaluations concerning products of exponential and periodic functions. Riv. Mat. Univ. Parma, 5, 1954, 63-81.-(b) on boundedness of the solutions of linear differential systems with periodic coefficients. Riv. Mat. Univ. Parma 5, 1954, 137-167.(c) Periodic solutions of nonlinear systems of differential equations. Riv. Mat. Univ. Parma 5, 1954, 281-311.-(d) On a class of linear differential equations with periodic coefficients. Illinois J. Math. 1, 1957, 98-104.-(e) Linear systems of first and second order differential equations with periodic coefficients. Illinois J. Math. 2, 1958, 586591.-(f) Sufficient conditions for the existence of periodic solutions of systems of weakly nonlinear first and second order differential equations. Journ. Math. Mech. 7, 1958, 163-172.-(g) A short proof of a boundedness theorem for linear differential systems with periodic coefficients. Archive Rat. Mech. Anal. 2, 1959, 429-434.-(h) On the behavior of the solutions of linear periodic differential systems near resonance points. Contributions to the theory of nonlinear differential equations, 5, 1960, 55-90.-(i) On the stability of periodic solutions of weakly nonlinear periodic and autonomous differential 
systems. Contributions to the theory of nonlinear differential equations 5, 1960, 91-114.-(j) On the characteristic exponents of linear periodic differential systems. Boletin Soc. Mat. Mexicana, 1960.(k) Oscillations in Nonlinear Systems. McGraw-Hill 1963.-(l) Periodic solutions of a class of hyperbolic equations, Arch. Rat. Mech. Anal. 23, 1967, 380-398.-(m) Ordinary Differential Equations. Wiley-Interscience 1969.

[13] J. K. Hale, S. Bancroft, and D. Sweet, Alternative problems for nonlinear functional equations. J. Diff. Equations 4, 1968, 40-56.

[' '] W. S. Hall, Periodic solutions of a class of weakly nonlinear evolution equations. Archive Rat. Mech. Anal. To appear.

[15] W. A. Harris, Y. Sibuya, and L. Weinberg, Holomorphic solutions of linear differential systems at singular points. Archive Rat. Mech. Anal. 35, $1969,245-248$.

[16] W. A. Harris, Holomorphic solutions of nonlinear differential equations at singular points. SIAM Studies in Applied Mathematics 5, 1969, 184187.

[17] C. Imaz, Sobre ecuacious differenciales lineales periodicas con un parametro pequeno. Bol. Soc. Mat. Mexicana (2) 6, 1961, 19-51.

[18] H. W. Knobloch, (a) Remarks on a paper of Cesari on functional analysis and nonlinear differential equations. Michigan Math. J. 10, 1963, 417430.-(b) Eine neue Methode zur Approximation von periodischen Loesungen nicht linear Differentialgleichungen zweiter Ordnung. Math. Zeit. 82, 1963, 177-197.-(c) Comparison theorems for nonlinear second order differential equations. J. Diff. Equations 1, 1965, 1-25.

[19] E. M. Landesman and A. C. Lazer, Nonlinear perturbations of linear elliptic boundary value problems at resonance. Journ. Math. Mech. 19, $1970,609-623$.

[20] J. Locker (a) An existence analysis for nonlinear equations in Hilbert space. Trans. Amer. Math. Soc. 128, 1967, 403-413.-(b) An existence analysis for nonlinear boundary value problems. SIAM J. Appl. Math. $18,1970$.

[21] J. Mawhin, (a) Application directe de la méthode de Cesari a l'étude des solutions périodiques de systèmes différentiels faiblement non linéaires, Bull. Soc. Roy. Sci. Lieg̀e 36, 1967, 193-210.-(b) Solutions périodiques de systèmes différentiels faiblement non linéaires. Ibid., 36, 1967, 491-499.-(c) Familles de solutions périodiques das les systèmes différentils faiblement non linéaires. Ibid. 36, 1967, 500-509.-(d) Degré topologique et solutions périodiques des systemes différentiels non linéaires. Ibid. 38, 1969, 308-398. 
[22] A. Naparstek, (a) Composition of functions in certain Sobolev spaces. To appear.-(b) Periodic solutions of weakly nonlinear wave equations in Sobolev spaces. To appear.-(c) On the Cesari method and periodic perturbation problem for certain hyperbolic equations. To appear.

[23] C. Perello, A note on periodic solutions of nonlinear differential equations with time lags. Differential Equations and Dynamical Systems (J. P. LaSalle, ed.), Academic Press 1967, 185-188.

[24] D. Petrovanu (a) Solutions peŕriodiques pour certaines équations hyperboliques, Analele Stintifice Iasi, 14, 1968, 327-357.-(b) Periodic solutions of the Tricomi problem. Michigan Math. J. 16, 1969, 331-348.

[25] A. M. Rodionov, Periodic solutions of nonlinear differential equations with time lag. Trudy Seminar Differential Equations Lumumba University, Moscow 2, 1963, 200-207 (Russian).

[26] C. D. Stocking, Nonlinear boundary value problems in a circle and related questions on Bessel functions. Thesis, University of Michigan, 1970.

[27] S. A. Williams, A connection between the Cesari and Leray-Schauder methods. Michigan Math. J. 15, 1968, 441-448.

\section{B. OTHER REFERENCES}

[28] L. Hormander, Linear Partial Differential Operators, 3rd Ed., Springer 1969.

[29] J. Leray and J. Schauder, Topologie et équations fonctionelles, Ann. Sci. École Norm. Sup. 51, 1934, 45-78.

[30] L. A. Liusternik and V. J. Sobolev, Elements of functional analysis. Ungar 1961.

[31] L. V. Ovcjannikov, A singular operator in a scale of Banach spaces, Soviet Math. Doklady, 163, 1965, 1025-1028.

[32] I. G. Petrovsky, Lectures on partial differential equations. Interscience 1954.

[33] F. Treves, Ovcjannikov theorem and hyperdifferential operators. Inst. Mat. Pura Appl., Rio de Janeiro 1968. 


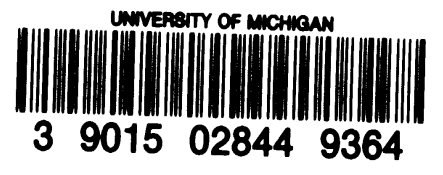

